\section{ENGLISH SOCIAL STRATIFICATION}

GOCIAL stratification exists in most societies, yet $\checkmark$ upon the English it exerts a powerful, subtle and complex influence, to a much greater extent than in younger culture-patterns. Strangely, however, the subject has attracted very little attention from psychologists. Psychologists ought to regard social stratification as a problem to be studied as objectively as a geologist studies the layers in a cliff face. The practical issues are of importance for the welfare of the future*.

Social stratification, however, is not the result of inevitable forces. Already since the War we have seen many changes, changes which are much more difficult to study in a large town than in a village. One classification distinguishes between those with opportunities and those without; in some parts of the country even the probability of living is less than in other more favoured localities, while positions of power and responsibility tend to go to persons chosen from an arbitrarily restricted group. If the diplomatic and civil services are to be made effectively democratic a definite study ought to be made of the requirements, and of the arguments for and against drawing from a narrow group.

Another classification is the social one based mainly on titles, money, ownership of land, education, contrasted with the stigma attached to the performance of manual work. This two-class division of English society means that public life is administered too frequently by people who know little at first hand about the life of the general public. Social differences are shown by choice of words and phrases, speechmelody, manners and games, and, to a certain extent, religion. The solution of many problems in social psychology needs more knowledge of the sentiments and ideals which characterize different social strata, so it is necessary to study the more and the less desirable social phenomena, to find out how, when the causes are not economic, they have arisen. It would be a pity if the lessening of privilege led to a decreasing tolerance of individual differences.

" "Psychological Aspects of English Social Stratiflcation." By T. H. Pear. Reprinted from the "Bull. John Rylands Library", 26, No. 2 1942 .

\section{DETERMINATION OF EQUILIBRIUM DIAGRAMS BY X-RAY METHODS}

$\triangle$ DISCUSSION on the determination of equiliA brium diagrams by $\mathrm{X}$-ray methods arranged by the Institute of Physics was held at the Royal Institution, London, on September 22. The chairman, Sir Lawrence Bragg, pointed out that the meeting was largely due to Dr. W. Hume-Rothery's initiative, and that it might be regarded as a continuation of the Institute's conference on X-ray technique held at Cambridge last Easter.

The opening talk was by Prof. E. A. Owen, Bangor, who was one of the first to apply X-ray methods to the study of equilibrium diagrams in an exact way, his subject being "The Determination of Phase Boundaries in Equilibrium Diagrams by X-Ray Methods". The accurate determination of lattice parameters was begun at the National Physical Laboratory, the initial publication being that of Preston and Gayler on the "Age Hardening of Copper-Aluminium", who used a focusing camera. This method has been largely used by Prof. Owen for determining phase boundaries. One of his first publications was "A Determination of the Limit of the $\alpha$-Solid Solution in the Copper-Zine System". The results agreed well with those found by the standard metallurgical methods, but copper-cadmium caused more trouble on account of the volatilization of the cadmium. Zinc alloys could also be made by heating a mixture of the powdered metals to the melting point of zinc. Then alloys were investigated in a high-temperature camera, the aluminium-zinc system being studied by this means. Later work by Gayler has confirmed the accuracy of this research, satisfactory results being obtained up to the melting point of zinc. The accuracy of the work depends on the composition and on the lattice parameter. The more rapidly the parameter changes with composition the better the results are likely to be. It is more difficult if the boundary changes very rapidly with increasing temperature, as in the copper-indium system, and accurate temperature control is vital.

In order to make alloys of more reliable composition, Prof. Owen has devised a shaking furnace. The materials are placed in small silica tubes which may be lined with graphite. After shaking for a quarter of an hour or longer, the alloy may be quenched or slowly cooled to obtain very small ingots down to half a gram in weight. Specimens selected from different parts of the ingots were sent away for analysis. There is usually agreement to $0 \cdot 1$ per cent with the intended compositions. The powders from these specimens are sieved before use and heat. treated in an evacuated silica, or 'Pyrex' tube. The work has been checked up by a satisfactory comparison of the results from alloys made up by fusion and interdiffusing. Identical parameter measurements have been obtained from powders and from solid specimens.

Extremely reliable results have been obtained even with the silver-arsenic system, no errors due to volatilization being detectable. A very important point is the effectiveness of quenching. A special furnace has been devised by means of which specimens may be quenched in less than two seconds.

The $\alpha$ boundary of the copper-tin system has been determined with an accuracy of 0.1 per cent. Similar results were found for the copper-aluminium system, but there is more difficulty with the silver-aluminium system because the $\beta$-phase is not retained, the possible error being 0.4 atomic per cent.

Dr. W. Hume-Rothery spoke on the application of X-ray methods to the determination of phase boundaries in metallurgical equilibrium diagrams. Two types of investigation are valuable: either the determination of a rough equilibrium diagram or the determination of an accurate diagram. For the rough survey it is preferable to use X-rays in conjunction with the older methods, the former for the lower, the latter for the higher, temperatures. There is, however, a possibility that the filings will not behave in the same way as the lumps, particular precautions being required to deal with two-phase alloys, where the powders may give misleading results unless each particle contains both phases in the same proportions.

For accurate work the liquidus and solidus are best determined by the older methods, but for investigating transformations in the solid state the hightemperature camera supersedes thermal analysis provided that the composition of the filings is accurately known. Moreover, the X-ray method is 